\title{
Gastrointestinal Stromal Tumor by AJCC v8 Stage
}

National Cancer Institute

\section{Source}

National Cancer Institute. Gastrointestinal Stromal T umor by A/CC v8 Stage. NCI

Thesaurus. Code C136767.

A term that refers to the staging of gastrointestinal stromal tumor (GIST), following the rules of the TNM AJCC V8 classification system. This staging system does not apply to pediatric GIST, familial GIST (germline mutant KIT or PDGFRA), or syndromic GIST (no AJCC staging system available). (from AJCC 8th Ed.) 